\title{
Abnormal fractional anisotropy in hydrocephalic children: a DTI
} study

\author{
Weihong Yuan*1, Ellen Air ${ }^{2}$, Mekibib Altaye ${ }^{1}$, Scott Holland ${ }^{1}$, Blaise Jones ${ }^{3}$, \\ Karin Bierbrauer ${ }^{2}$ and Francesco Mangano ${ }^{2}$
}

\begin{abstract}
Address: ${ }^{1}$ Paediatric Neuroimaging Research Consortium, Dept. of Radiology, Cincinnati Children's Hospital Medical Centre, 3333 Burnet Ave., Cincinnati, Ohio, 45229, USA, ${ }^{2}$ Division of Pediatric Neurosurgery, Cincinnati Children's Hospital Medical Centre, 3333 Burnet Ave., Cincinnati, Ohio, 45229, USA and ' ${ }^{2}$ Dept. of Radiology, Cincinnati Children's Hospital Medical Centre, 3333 Burnet Ave., Cincinnati, Ohio, 45229, USA

* Corresponding author
\end{abstract}

from 52nd Annual Meeting of the Society for Research into Hydrocephalus and Spina Bifida Providence, RI, USA. II-I 4 June 2008

Published: 3 February 2009

Cerebrospinal Fluid Research 2009, 6(Suppl I):SI4 doi:I0.II86/I743-8454-6-SI-SI4

This abstract is available from: http://www.cerebrospinalfluidresearch.com/content/6/SI/SI 4

(C) 2009 Yuan et al; licensee BioMed Central Ltd.

\section{Background}

Diffusion tensor imaging (DTI) is an advanced neuroimaging technique that characterizes in vivo anisotropic diffusion in the white matter (WM). However, its application in hydrocephalus patients, especially in those at very young age, is very limited. The purpose of this study is to apply DTI to test the abnormality of anisotropic diffusion properties at various WM regions in hydrocephalic children.

\section{Materials and methods}

We retrospectively reviewed clinical DTI datasets and identified three groups of participants. The control group included 61 patients (Age range $=0.2-206.0$ months, Mean $\pm S D=110.3 \pm 65.3$ months $)$ who were scanned for non-CNS related problems and had no prior record of a neurological disorder. The pre-shunt hydrocephalus group included 12 patients (Age range $=0.03-78.15$ months, Mean $\pm \mathrm{SD}=8.68 \pm 19.06$ months) who had MRI/DTI prior to shunt surgery. The post-shunt hydrocephalus group included 19 patients (Age range $=12.76-$ 212.65 months, Mean $\pm \mathrm{SD}=59.97 \pm 62.42$ months $)$ who had MRI/DTI after shunt surgery. Fractional anisotropy (FA) was computed in selected region of interest in corpus callusom and internal capsule.

\section{Results}

Consistent to the published literature, FA values of normal children in all the ROIs demonstrated a characteristic exponential pattern during development. In general, the FA values undergo a very rapid rise during the first 12 months of life. It begins to level off in the second year and completes by 36 months of age.

In genu of corpus callosum (gCC), only $1 / 11$ patients in the pre-shunt group had normal FA value while all other patients $10 / 11$ had abnormally low FA value. On the other hand, for patients in post-shunt group, 11/18 had normal FA and only $7 / 18$ had abnormally low FA. The frequency of occurrence of low FA is significantly different ( $p$ $=0.007$, Fisher's exact test) in the two groups. In posterior limb of internal capsule (PLIC), 7/12 had normal FA and $5 / 12$ had abnormally high FA in the pre-shunt group. In post-shunt group, the percentage of patients with normal FA $(11 / 19,58.9 \%)$ is similar to the pre-shunt group. However, the rest of the patients (8/19) all had abnormally low FA.

\section{Conclusion}

This study demonstrated the efficacy of applying DTI in investigating WM integrity in paediatric hydrocephalic patients at early childhood. The FA abnormality and its difference in frequency of occurrence or the direction of deviation from normal range between the two hydrocephalic groups may bear significant implication for the underlying injury mechanism as well as the reversibility at various WM regions. 